\title{
A Transformation Group and Its Subgroups
}

\author{
Euich Miztani \\ JEIN Institute for Fundamental Science \\ 5-14, Yoshida-honmachi, Sakyo-ku, Kyoto, 606-8317, Japan \\ Lecturer, Aikodai Meiden High School \\ 3-2-12, Wakamizu, Chikusa-ku, Nagoya, 464-8540, Japan
}

\begin{abstract}
We discuss a transformation as follows. There is a point on the number line, then we move it on an integer $m$ to another one $n$ on the number line. We denote the transformation with ${ }_{m} T_{n}$. We think of all the elements $\left.G={ }_{m} T_{n} \mid m, n \in \mathrm{Z}\right\}$. Then, we discuss the binary operation ${ }_{l} T_{n}=T_{m} T_{m} T_{n}$. It denotes the repetition of transformation. $G$ makes a group under the binary operation. This is a modeling of group in [1] on the number line. In this paper, we think of the subgroups.

2010 Mathematics Subject Classification. 20A10
\end{abstract}

\section{Introduction}

First of all, as shown in Figure 1, we think of a point on the number line, then we move it on the integer $m$ to the other one $n$ on the number line. We denote the transformation with ${ }_{m} T_{n}$. 


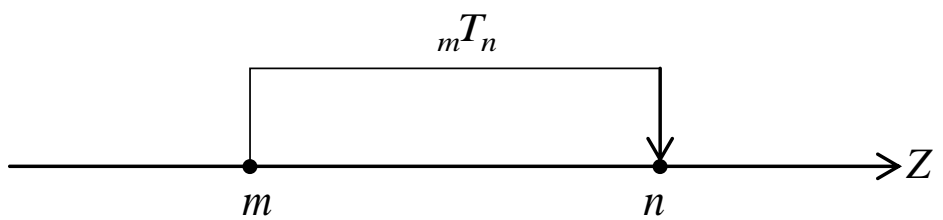

Fig.1

We think of all the elements in $G=\left\{_{m} T_{n} \mid m, n \in Z\right\}(*)$. Now, let us think of the binary operation ${ }_{l} T_{m m} T_{n}={ }_{l} T_{n}(* *)$ for the repetition of transformation. It denotes a series of transformations of a point on an integer $l$ to $m$, then $m$ to $n$ on the number line. There are 6 types of transformations for ${ }_{i} T_{n}={ }_{l} T_{m m} T_{n}$ as shown in Figure 2, it is clear that any types of transformation hold the binary operation closed in $G$.
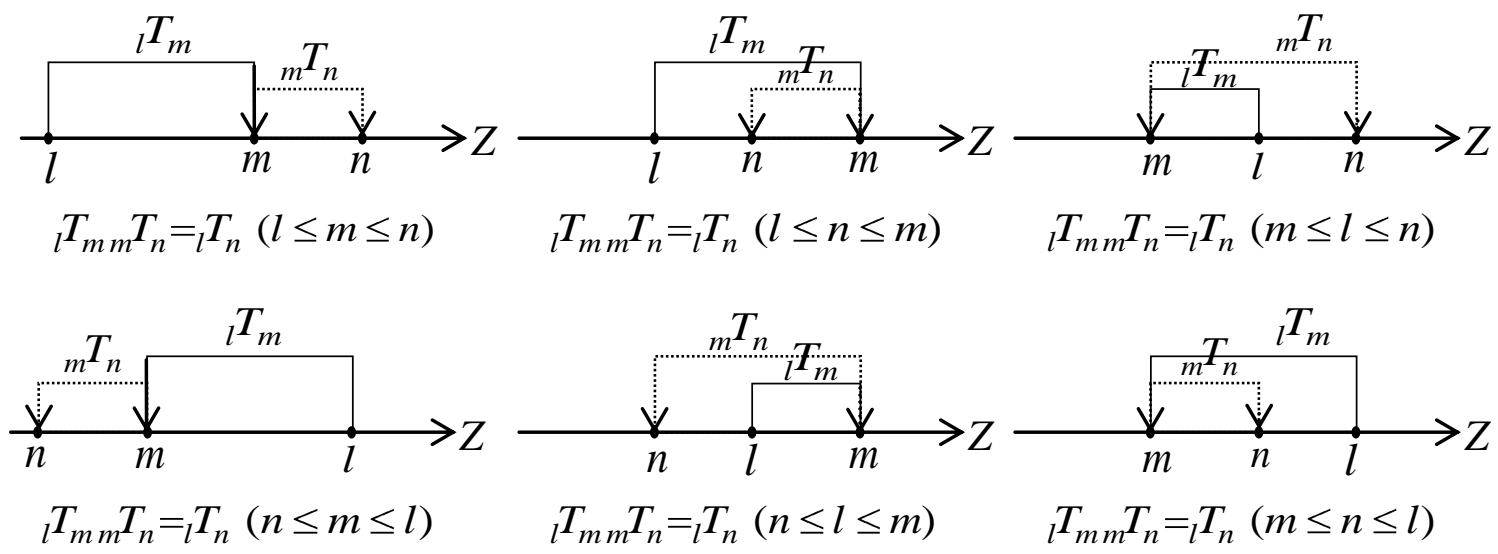

Fig.2

Theorem 1.1. For any $i, j, k$ and $l$ in $Z$, laws under the binary operation rule in $G$ are as follows.
(1.1) ${ }_{i} T_{j} T_{k}={ }_{i} T_{k}$
(1.2) $\quad\left({ }_{i} T_{j j} T_{k}\right)_{k} T_{l}={ }_{i} T_{j}\left({ }_{j} T_{k} T_{l}\right)$
(1.3) $\quad\left({ }_{i} T_{j}\right)^{-1}={ }_{j} T_{i}$
(1.4) ${ }_{i} T_{j j} T_{i}={ }_{j} T_{i} T_{j}=T_{i} T_{i}={ }_{j} T_{j}=I$

Proof. (1.1) is obvious by the definition of binary operation. (1.2): From (1.1), $\left.{ }_{(} T_{j} T_{k}\right)_{k} T_{l}=T_{k} T_{k} T_{l}=T_{i},{ }_{i} T_{j}\left({ }_{j} T_{k k} T_{l}\right)={ }_{i} T_{j} T_{l}={ }_{i} T_{l}$. (1.3) is clear under the operation on 
the number line. (1.4): Since ${ }_{i} T_{i}$ and ${ }_{j} T_{j}$ are "mapping to itself" on the number line, ${ }_{i} T_{i}$ or ${ }_{j} T_{j}$ denotes the identity transformation(: in other words, since they are equivalent with $I\left(:_{i} T_{i i} T_{j}=I T_{j}=T_{i}=T_{i} T_{j} T_{j}=T_{i} T_{j} I=T_{j}\right)$, the identity is unique.) Thus, $G$ makes a group.

\section{The Subgroups}

Now, let us think of the subgroups. They mainly consist of two subgroups $H=\left\{{ }_{2 m-1} T_{2 n-1} \mid m, n \in \mathrm{Z}\right\}$ and $H^{\prime}=\left\{{ }_{2 m} T_{2 n} \mid m, n \in \mathrm{Z}\right\}$. The rest $G-\left(H \cup H^{\prime}\right)=\left\{_{2 i-1} T_{2 j}{ }_{2 k} T_{2 l-1} \mid i, j, k, l \in \mathrm{Z}\right\}$ is not a subgroup but subset.

Remark. $\left\{{ }_{2 m-1} T_{2 n}\right\}\left\{{ }_{2 n} T_{2 k-1}\right\}=\left\{{ }_{2 m-1} T_{2 k-1}\right\}=H$ and $\left\{{ }_{2 m} T_{2 n-1}\right\}\left\{{ }_{2 n-1} T_{2 k}\right\}=\left\{{ }_{2 m} T_{2 k}\right\}=H^{\prime}$.

We actually need to pay attention to such operations. If we take the elements like ${ }_{4} T_{3} T_{2}={ }_{4} T_{2}$, it is not in $G-\left(H \cup H^{\prime}\right)$ but in $H^{\prime}$. These operations are equivalent with in $G$. Eventually, $H, H^{\prime}$, and $G-\left(H \cup H^{\prime}\right)$ are disjoint. The direct sum decomposition is, $\therefore G=\left\{{ }_{2 m-1} T_{2 n-1}\right\} \amalg\left\{{ }_{2 m} T_{2 n}\right\} \amalg\left\{{ }_{2 i-1} T_{2 j}\right\} \amalg\left\{{ }_{2 k} T_{2 l-1}\right\}$.

Proposition 2.1. $H$ and $H^{\prime}$ are not normal subgroups of $G$.

Proof. Let us assume that $H$ is the normal subgroup. Let $g$, for example, be any element in $\left\{_{2 m-1} T_{2 n}\right\}$. Operating $g H$, it is impossible to operate unless any element in $H$ is ${ }_{2 n} T_{2 l-1}$ or ${ }_{2 n} T_{2 l}$ under the binary operation rule. Therefore, $g H$ cannot be the left coset. $\therefore g H \notin G$ in this case. Operating $H g$, because of $H=\left\{_{2 k-1} T_{2 m-1}\right\}$, it holds and the right coset is $H g=\left\{{ }_{2 k-1} T_{2 n}\right\} . \therefore g H \neq H g$. Likewise, let us assume that $H^{\prime}$ is the normal subgroup. For $\forall g^{\prime} \in\left\{{ }_{2 m} T_{2 n-1}\right\}$, operating $g^{\prime} H^{\prime}$, then it cannot be operated unless any elements in $H^{\prime}$ are $\left\{{ }_{2 n-1} T_{2 l}\right\}$ or $\left\{_{2 n-1} T_{2 l-1}\right\}$. They are not in $H^{\prime}$ either. $\therefore g^{\prime} H^{\prime} \notin G$. Operating $H^{\prime} g^{\prime}, H^{\prime} g^{\prime}$ holds since any element in $H^{\prime}$ are $\left\{_{2 k} T_{2 m}\right\}$. Then, $H^{\prime} g^{\prime}=\left\{\left\{_{2 k} T_{2 n-1}\right\} . \therefore g^{\prime} H^{\prime} \neq H^{\prime} g^{\prime}\right.$.

As another way of taking of the subgroups, let us think of $H^{\prime \prime}=\left\{{ }_{\text {ln }} T_{\mathrm{ln}} \mid l, m, n \in \mathrm{Z}\right\}$. However, none of them is the normal subgroup. 
Proof. For $H^{\prime \prime}\left(=H_{l}\right)$, only if $l=1$, then $g^{\prime \prime} H^{\prime \prime}=H^{\prime \prime} g^{\prime \prime}$ for $\forall g^{\prime \prime} \in\left\{\left\{_{m} T_{1}\right\}\left(\right.\right.$ or $\forall g^{\prime \prime}=\left\{\left\{_{1} T_{m}\right\}\right)$. ${ }_{m} T_{1}$ (or ${ }_{1} T_{m}$ ) denotes element transforming 1 to $m$ (or $m$ to 1 ) on the number line. $\therefore g G=G g$.

Proposition 2.2. The maximal proper subgroup $H^{\prime \prime \prime}$ is a set extracted elements whose either index number is fixed and all the inverses (: any element should have inverse also in subgroup by two-step subgroup test) from $G$ like, for example, $H^{\prime \prime}=G-\left(\left\{_{m} T_{0}\right\} \bigcup\left\{{ }_{0} T_{m}\right\}\right)$. It graphically means only the point $\{0\}$ is skipped in a series of transformation on the number line. Likewise, it is not the normal subgroup also.

Proof. ${ }_{m} T_{0} H^{\prime \prime \prime}$ does not hold but $H^{m{ }_{m}} T_{0}$ does. $\quad \therefore_{m} T_{0} H^{\prime \prime \prime} \neq H^{\prime \prime \prime}{ }_{m} T_{0}$.

From this proof, it is clear that any other smaller subgroups extracted any other subsets or the families of sets will not be normal. The simplest example is a case of $G=\left\{{ }_{m} T_{n} \mid m, n=0,1,2\right\}$ and the smallest subgroups (: except for the identity as subgroup) are $\left\{{ }_{m} T_{n} \mid m, n=0,1\right\},\left\{{ }_{m} T_{n} \mid m, n=1,2\right\}$, and $\left\{{ }_{m} T_{n} \mid m, n=0,2\right\}$. It is trivial that none of these subgroups is normal based on the proof above.

Contrarily, by the way, what will happen if we sum up those extracted families of sets? What we get interested in will be if such a summed-up subset makes a subgroup of $G$. To confirm it, let us think of such a total subset $s_{k}$ as follows.

$s_{k}=\left(\left\{_{m} T_{-|m|}\right\} \bigcup\left\{\left\{_{|m|} T_{m}\right\}\right) \bigcup \ldots \bigcup\left(\left\{_{m} T_{-1}\right\} \bigcup\left\{{ }_{-1} T_{m}\right\}\right) \bigcup\left(\left\{_{m} T_{0}\right\} \bigcup\left\{\left\{_{0} T_{m}\right\}\right) \bigcup\left\{{ }_{m} T_{1}\right\} \bigcup\left\{T_{1} T_{m}\right\} \bigcup \ldots \bigcup\left\{\left\{_{m} T_{k}\right\} \bigcup\left\{{ }_{k} T_{m}\right\}\right)\right.\right.$, where $k \leq m$. If $k=m$, then $s_{k}=s_{m}=G$. If thinking of the binary operation ${ }_{k+1} T_{k k} T_{k+2}, \quad{ }_{k+1} T_{k k} T_{k+2}={ }_{k+1} T_{k+2} \notin s_{k}$. Besides, if ${ }_{m} T_{m-1-1} T_{m},{ }_{m} T_{m-1 m-1} T_{m}={ }_{m} T_{m} \notin S_{m-1}$. Thus, any of such total subsets is not the subgroup. If adding ${ }_{m} T_{m}$ on to $s_{m-1}$, $s_{m-1} \amalg_{m} T_{m}$ results in $G$. Verifying them on the number line, it will be clearer. 
Now, we condition that any subgroup assures to transform to any number in its arithmetic sequence on the number line. Let us consider the subgroups $H$ as $G^{\prime}$ and $H^{\prime}$ as $G^{\prime \prime}: G^{\prime}$ and $G^{\prime \prime}$ are respectively independent from $G$.

Corollary 2.3. The normal subgroup of $G^{\prime}$ is $G^{\prime}$ itself, $G^{\prime \prime}$ is $G^{\prime \prime}$ as well.

Proof. $G^{\prime}$ : Since subgroups of $G^{\prime}$ including itself are $\left\{_{2 m-1} T_{2 n-1}\right\},\left\{_{4 m-1} T_{4 n-1}\right\}$, $\left\{{ }_{6 m-1} T_{6 n-1}\right\}, \ldots$, and $\left\{_{p \backslash 2\}} T_{q \backslash\{2\}}\right\}$, where " $p \backslash\{2\}$ " and “ $q \backslash\{2\}$ " are any prime numbers except for initial prime $\{2\}$ (, and we accept negative prime numbers except for $\{-2\}$ on the number line,) then $G^{\prime}$ is uniquely possible to operate with any $g^{\prime}$ in $\left\{{ }_{2 m-1} T_{1}\right\}$ or $\left\{T_{2 n-1}\right\} . \therefore g^{\prime} G^{\prime}=G^{\prime} g^{\prime}$.

$G$ ": Subgroups of $G$ "including itself are $\left\{{ }_{2 m} T_{2 n}\right\},\left\{_{4 m} T_{4 n}\right\},\left\{{ }_{6 m} T_{6 n}\right\}, \ldots$. Therefore, only $G^{\prime \prime}$ is possible to operate with $\forall g^{\prime \prime} \in\left\{\left\{_{2 m} T_{2}\right\}\right.$ or $\forall g^{\prime \prime} \in\left\{{ }_{2} T_{2 n}\right\} . \quad \therefore g^{\prime \prime} G^{\prime \prime}=G^{\prime \prime} g^{\prime \prime}$.

Corollary 2.4. Considering $G_{3}=\left\{\left\{_{4 m-1} T_{4 n-1}\right\}, \quad G_{5}=\left\{\left\{_{6 m-1} T_{6 n-1}\right\}, \quad G_{7}=\left\{\left\{_{8 m-1} T_{8 n-1}\right\}, \ldots\right.\right.\right.$, and $G_{p}=\left\{{ }_{p} T_{q}\right\}$ (or $G_{p}=\left\{\left\{_{p \nmid\{2\}} T_{q \backslash\{2\}}\right\}\right.$ ) independent from $G^{\prime}$, the normal subgroup is itself.

Proof. Subgroups of $G_{3}$ including itself are $\left\{{ }_{4 m-1} T_{4 n-1}\right\},\left\{{ }_{8 m-1} T_{8 n-1}\right\},\left\{\left\{_{12 m-1} T_{12 n-1}\right\}, \ldots\right.$. Therefore, $G_{3}$ is uniquely possible to operate with $\forall g_{3} \in\left\{\left\{_{4 m-1} T_{3}\right\}\right.$ or $\forall \mathrm{g}_{4}=\left\{{ }_{3} T_{4 n-1}\right\}$. $\therefore g_{4} G_{4}=G_{4} g_{4}$. Identically, subgroups of $G_{5}$ including itself are $\left\{{ }_{6 m-1} T_{6 n-1}\right\}$, $\left.\left\{{ }_{12 m-1} T_{12 n-1}\right\}, \quad{ }_{18 m-1} T_{18 n-1}\right\}, \ldots$ Thus, only $G_{5}$ is possible to operate with $\forall g_{5} \in\left\{{ }_{6 m-1} T_{5}\right\}$ or $\forall g_{5} \in\left\{{ }_{5} T_{6 n-1}\right\} . \quad \therefore g_{5} G_{5}=G_{5} g_{5}$. Algorithmically, proofs of $G_{7}=\left\{\left\{_{8 m-1} T_{8 n-1}\right\}\right.$, $G_{9}=\left\{{ }_{10 m-1} T_{10 n-1}\right\}, G_{11}=\left\{{ }_{12 m-1} T_{12 n-1}\right\}, \ldots$, and $G_{p}$ are same as above.

If taking into account the Green-Tao theorem, there are still arithmetic sequences consisting only of prime numbers. So that, there are elements of subgroups transforming on such the prime arithmetic sequences on the number line. Such a set of elements naturally makes a subgroup of $G_{p}$. If considering it as a group $G_{p}^{\prime}$ independent from $G_{p}$, proof of $G_{p}^{\prime}$ is simple is 
same as above. However, it will be argumentative in a way. Even if considering it as a subgroup, it is therefore clear that it is not the normal subgroup of $G_{p}$.

Corollary 2.5. $G_{4}=\left\{{ }_{4 m} T_{4 n}\right\}, G_{6}=\left\{{ }_{6 m} T_{6 n}\right\}, G_{8}=\left\{{ }_{8 m} T_{8 n}\right\}, \ldots$ independent from $G$ " are their normal subgroups are themselves.

Proof. Subgroups of $G_{4}$ including itself are $\left\{{ }_{4 m} T_{4 n}\right\},\left\{\left\{_{8 m} T_{8 n}\right\}, \quad\left\{{ }_{12 m} T_{12 n}\right\}, \ldots\right.$. Therefore, $G_{4}$ is uniquely possible to operate with $\forall g_{4} \in\left\{{ }_{4 m} T_{4}\right\}$ or $\forall \mathrm{g}_{4}=\left\{{ }_{4} T_{4 n}\right\}$. $\therefore g_{4} G_{4}=G_{4} g_{4}$. Identically, subgroups of $G_{6}$ including itself are $\left\{_{6 m} T_{6 n}\right\},\left\{{ }_{12 m} T_{12 n}\right\}$, $\left\{{ }_{18 m} T_{18 n}\right\}, \ldots$. Thus, only $G_{6}$ is possible to operate with $\forall g_{6} \in\left\{{ }_{6 m} T_{6}\right\}$ or $\forall \forall g_{6} \in\left\{{ }_{6} T_{6 n}\right\}$. $\therefore g_{6} G_{6}=G_{6} g_{6}$. Algorithmically, proofs of $G_{8}=\left\{\left\{_{8 m} T_{8 n}\right\}, G_{10}=\left\{{ }_{10 m} T_{10 n}\right\}, G_{12}=\left\{{ }_{12 m} T_{12 n}\right\}, \ldots\right.$ are same as above.

\section{Notation}

1. If extending the domain $\mathbf{Z}$ of (*) to $\mathbf{R}$ and introducing a Cartesian product such with $G=\left\{T_{i} T_{j} \mid i, j \in \mathrm{R}\right\}$ and $G^{\prime}=\left\{{ }_{k} S_{l} \mid k, l \in \mathrm{R}\right\}$, then we could define the Cartesian product of mappings as follows.

$R \times R \rightarrow R \times R$

$(i, k) \mapsto T(i, k)=\left({ }_{i} T_{j}(i){ }_{k} S_{l}(k)\right)=(j, l)$

2. A reader may feel that the binary operation $(* *)$ is partially defined, not for any two elements arbitrarily taken from $G$. However, we have to take notice of that group axioms do not claim such a whole process should be done. To confirm it, let us try to give five conditions as the group axioms as follows.

(1). We randomly take any two elements in a set $G$.

(2). For any two elements taken from $G$, the binary operation is closed in $G$, such that for any $a, b, c$ in $G, a b=c$.

(3). For any $a, b, c$ in $G,(a b)_{c}=a(b c)$ : associative law holds.

(4). There exists unique identity $e$.

(5). For each $a$ in $G$, its inverse $b$ exists such that $a b=b a=e$. 
What we have to pay attention to is whether the first condition should be included in the group axioms. If accepting it, we should introduce a concept of axiom in probability theory. That is, in group theory, we suppose the whole event for any two elements (: two elementary events) arbitrarily taken from $G$ in the manner of probability theory, then define binary operation such for any elements taken from $G$ at random. In other words, we should consider so called measure theory for probability in group theory. Group axioms naturally do not claim such a process in probability theory.

In fact, in linear algebra, for any $k-b^{-} l, l-b^{-} m$, and $m-b^{-} n$ matrices $M_{k l}, M_{l m}$, and $M_{m n}$ in a set of matrices $M$, binary operation is defined, and then the associative law holds: $\left(M_{k l} M_{l m}\right) M_{m n}=M_{k l}\left(M_{l m} M_{m n}\right)$. In summary,

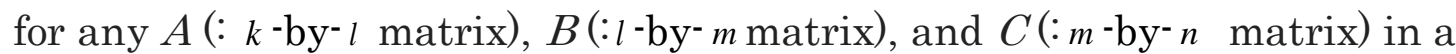
set $S$, binary operation is defined, then, $(A B) C=A(B C)$. However, it does not follow the first condition above. Thus, associative law (: as one of group axioms) is purely defined without any concept of probability theory belonging to the other axiomatic system.

\section{References}

[1] Euich Miztani, Projections and Dimensions, p. 7-18, Communications in Applied Geometry, Volume 1, 2011, Research India Publications. 
